\title{
Thermographical Analysis of Paper During Tensile Testing and Comparison to Digital Image Correlation
}

\author{
A. Hagman ${ }^{1}$ (D) M. Nygårds ${ }^{2}$
}

Received: 24 March 2016 / Accepted: 26 October 2016 / Published online: 16 December 2016

(C) The Author(s) 2016. This article is published with open access at Springerlink.com

\begin{abstract}
The thermal response in paper has been studied by thermography. It was observed that an inhomogeneous deformation pattern arose in the paper samples during tensile testing. In the plastic regime a pattern of warmer streaks could be observed in the samples. On the same samples digital image correlation (DIC) was used to study local strain fields. It was concluded that the heat patterns observed by thermography coincided with the deformation patterns observed by DIC. Because of its fibrous network structure, paper has an inhomogeneous micro-structure, which is called formation. It could be shown that the formation was the cause of the inhomogeneous deformations in paper. Finite element simulations was used to show how papers with different degrees of heterogeneity would deform. Creped papers, where the strain at break has been increased, were analysed. For these paper it was seen that an overlaid compaction of the paper was created during the creping process. During tensile testing this was recovered as the paper network structure was strained.
\end{abstract}

Keywords Paperboard - Elastic-plastic properties . In-plane heterogeneity · Thermography · Digital image correlation $\cdot$ Formation $\cdot$ Finite element method

A. Hagman

AntonHag@kth.se

1 BiMaC Innovation, Department of Solid Mechanics, School of Engineering Sciences, KTH Royal Institute of Technology, 10044 Stockholm, Sweden

2 BiMaC Innovation, INNVENTIA AB, Box 5604, 11486 Stockholm, Sweden

\section{Introduction}

Paper is a fibrous material that can be designed via the fibre orientation, and different degrees of anisotropy can be created. The fibres will predominately be orientated in the in-plane directions, and the degree of anisotropy in the inplane direction between the machine direction (MD) and the cross machine direction (CD) is often in the range of 1-5, while it can differ factor a 50-100 between the through thickness direction (ZD) and MD [1, 2]. Paper does however have an inhomogeneous structure because of formation effects, where the fibres get entangled in flocs, i.e. denser clusters of fibers [3]. This result in local density variations, which causes strength variations since the ability to form joints between the fibres depend on local contacts between the fibers. The exact nature of the bonds in the fiber-fiber joints is still debated, as well as how large the contact area between the fibers is. What can be concluded is that the joint strength distribution is usually skewed and can be described by a Weibull or exponential distribution, see e.g. [4, 5].

In many applications paper is engineered to have high strength. However, in order to compete with plastics it is also important that the strain at break can be engineered. Although paper has the advantage of being a sustainable material, there are still hurdles to overcome to be competitive against plastics. One such hurdle is the ability to form packages with rounded surfaces which is advantageous for appealing designs. One part of this is to produce paper and paperboard that have a high strain-ability. By utilization of creping the strain at break in MD can be altered, which is commonly used to make sack papers. When paper is creped it will be compacted in the MD, which will increase the strain at break, but also decrease the tensile strength and stiffness. 
Thermography has previously been used as a tool in paper research, e.g. [6-10], when studying damage and crack propagation as well as energy consumption during tensile loading of paper. It has also been used to study wet web properties [11]. As with many techniques, thermography has matured in recent years resulting in new powerful and more user friendly systems, e.g. such as those produced by FLIR $^{\circledR}$ Systems, Inc. This makes it a perfect time to re-assess the possibilities that such systems presents for paper research, and exploring the mechanisms behind the increased strain at break in creped papers is a good candidate for such a re-examination.

In previous studies, e.g. [7-10, 12], it has been shown that the average temperature of the sample during tensile loading has two clear phases. First the paper cools down to below the initial temperature, which is followed by an temperature increase well beyond the initial sample temperature. These two phases correspond well to the elastic and the non-elastic regions of the stress-strain curve. The initial decrease is attributed by Dumbleton et al. [13], using classical thermodynamics, to paper having a positive thermal expansion coefficient and Poisson ratios below 0.5. In most of these previous thermography studies the main interest has been focused on the rupture starting point, and to a lesser extent the crack path. This was partly due to the resolution of the available equipment at the time. In those studies it was observed that it is possible to identify the starting point for rupture, since there is a considerable temperature increase (some degrees centigrades) in the rupture area. Further it was also shown that the rupture path did not necessarily follow high temperature streaks [14]. This behaviour was explained by the large amount of elastic energy that is stored in the sample together with the changing load situation around the crack tip.

To further increase the understanding of both the thermal response of paper during straining as well as the deformation mechanisms at work in creped papers the intent is to combine the thermography with digital image correlation (DIC) measurements. DIC has been used together with thermography previously, but to the author's knowledge not on paper. Examples of such uses can be found in Chrysochoos et al. [15], where several examples are given, and more recently Cholewa et al. [16], where DIC is used together with thermography to analyse the behaviour of paint in high temperature.

Since paper is an heterogeneous material by nature, it is of interest to compare any patterns seen to the fibre distribution in the paper, i.e. the formation. Previous work on localized straining in paper, such as the work by Korteoja et al. [17] with silicone treated papers or the work by Hagman and Nygårds [18] with digital image correlation, DIC, has shown that the localization occurs in paper when it is stretched plastically. To enable straight forward comparisons between the formation, which was measured unstrained, and stained patterns, a finite element model based on the formation will be presented.

The aim of this work was to deepen the knowledge of paper response during straining. This will be done by comparing the results from thermography with results from DIC, the optical formation of the paper and finite element simulations.

\section{Materials and Methods}

Two different paper qualities were considered in this work. The papers were produced on a pilot paper machine; thereafter they were creped to alter the properties to different degrees. The papers were tested using several methods. Tensile testing, to acquire the overall tensile response of the papers. Thermography, to measure the thermal response of the papers. Digital image correlation, to measure the local strain behaviour, primarily in the load direction. Formation assessment to assess the basic heterogeneity. Finally a finite element analysis was performed to facilitate comparison between the formation and the thermal and local strain response, as well as investigate the possibility to predict the papers behaviour during tensile loading.

Papers Deformable papers have been produced on the FEX pilot paper machine at Innventia AB. In the trial unbleached chemical pulp fibres was used together with polyvinylamine (PVAm) as a strength additive. Papers with two different headbox pressures were produced; one with low pressure $(6.7 \mathrm{kPa})$ which should result in a low degree of anisotropy (E) and one with high pressure $(8.0 \mathrm{kPa})$ which should give high degree of anisotropy (F). By using this procedure, single ply papers with grammage $240 \mathrm{~g} / \mathrm{m}^{2}$ and a strain at break of $3 \%$ in MD and $3.5 \%$ in CD could be produced. Thereafter the strain at break could be increased using the off-line creping device.

Creping and drying A lab scale creping device has been used to increase the strain at break. It consists of a nip with a lower steel cylinder (diameter $200 \mathrm{~mm}$ ), and an upper rubber coated steel cylinder (steel diameter $200 \mathrm{~mm}$ and rubber thickness $10 \mathrm{~mm}$ ). During creping the rolls were run with a speed difference, where the lower steel roll had the highest speed. In this test, speed differences of 10, 15 and $20 \%$ were used. The samples will further on be labelled according to which material that was used, E or F, and the speed difference yielding samples E0, E10, E15, E20, and equally for paper F. When the papers had been produced they were taken from the FEX machine after the press sections, the dryness level was then about $40 \%$. Thereafter, the papers 
were put in sealed plastic bags and stored in a refrigerator until creping could be done, several days later. During the storing the moisture was maintained in the sheets. The wet sheets were run through the creping nip. Thereafter they were dried on a photo mount dryer at $70{ }^{\circ} \mathrm{C}$, this drying technique should be considered not to apply any constraints, and hence considered to be equal to free drying. As a comparison the sheets were also dried in the one cylinder dryer at FEX, which was considered to be restrained drying, the latter were labelled $\mathrm{E} 0 *$ and $\mathrm{F} 0 *$.

Tensile testing Uniaxial tensile tests were performed on two different tensile testers. The first set of thermographs were captured using an MTS hydraulic tensile tester with a $1 \mathrm{kN}$ loadcell located in Innventias climate controlled laboratory $\left(23{ }^{\circ} \mathrm{C}, \quad 50 \% \quad R H\right)$. The combined thermographs and DIC measurements were performed on a smaller servo electric tensile tester with a $2500 \mathrm{~N}$ load cell (Instron) located in a climate controlled room $\left(23^{\circ} \mathrm{C}, \quad 50 \% R H\right)$ at the Solid Mechanics laboratory at KTH. Before testing, different strain rates were evaluated to find a clear thermal signal, and also to be able to resolve the process taking place in the paperboard with both camera equipments. If the strain rate was too low, the smaller amount of emitted energy in combination with the dissipation in the paper made the thermal signal hard to interpret. While if the strain rate was to high, the strain measurements suffered. Good results could be achieved with a strain rate of $100 \% /$ minute, which was in accordance with the tensile test standard (ASTM-828). On both set ups the load, $F$, was measured by a load cell, and the piston displacement, $u$, was used to calculate strain. In order to detect deformation patterns in the specimen fairly large specimens, with width $b=50 \mathrm{~mm}$ and a distance $L=100 \mathrm{~mm}$ between the clamps were used. With a ratio of $L / b=2$ the lateral restraint from the clamps can affect the mechanical response of the sample, but previous work [18] indicates that the effect is limited to the ends of the sample, while the strain patterns emerge through out the whole sample. From the measurement stress was calculated as $\sigma=F / b / t$, where $t$ was the average paper thickness, and strain was calculated as $\varepsilon=u / L$. During testing both an IR camera and a digital image correlation system, one on each side of the sample, were used to study deformations in the paper. In this study one ply papers were used. The deformation on both sides of the sample will then be equal. If multi ply paper boards or other thicker paper grades would be considered, were the fibre flocs does not go through the whole thickness, this might not be possible. A sketch of the set up with both DIC and thermography can be found in Fig. 1. Thickness, grammage, strength and strain at break data from the papers can be found in Table 1 . Since the amount of data captured by both the thermographs and the DIC was rather

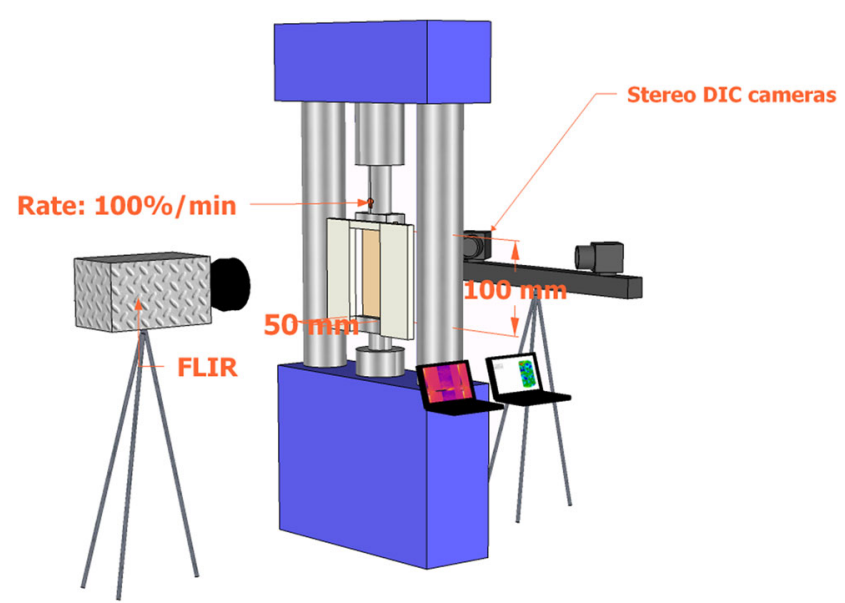

Fig. 1 A sketch of the set-up using both thermo camera and DIC

immense, the number of samples at each test point was limited to one or a few. These samples were carefully examined so that the measured stress-strain behaviour corresponded to the series of standard tests. Furthermore, the samples were examined such that they did not fail in the clamps or due to obvious flaws. Examples of stress-strain curves for the samples used in the thermographic measurements can be found in Fig. 2, from these it was clear that the paper show similar behaviour to those measured in standard testing even though the samples were wider. The standard tests were performed using an L\&W TH1 Alwetron (Lorentzen \& Wettre, Stockholm, Sweden) in accordance with the tensile test standard(ASTM-828).

In Fig. 2, it can be noted that the $\mathrm{E}$ and $\mathrm{F}$ samples were fairly similar, although paper $\mathrm{F}$ had a fairly higher degree of anisotropy compared to paper E. From the curves in Fig. 2, elastic modulus, failure stress and strain at break have been evaluated, these data and those from the standard tests are displayed in Table 1, values from Fig. 2 are within brackets. Creping had the effect that the strain at break was increased with higher degree of compaction. Unfortunately, the whole speed difference during creping could not be accounted for, which would indicate that there was slipping in the nip. The strain at break could however be increased to $10 \%$, which was higher than the strain at break in CD, cf. Table 1 . However, as the strain at break increased the elastic modulus and failure stress decreased. In Fig. 3 photos of a creped paper and a reference paper are compared. The main difference was that the fibres are freer in the reference paper than in the creped sample. Floc sizes and distributions were similar between the two papers.

IR camera For the thermographical analysis an infrared (IR) camera from FLIR ${ }^{\circledR}$ Systems, Inc., SC 6000 , was used. The camera has previously been used by Hyll et al. [6] 
Table 1 Measured properties of the papers

\begin{tabular}{|c|c|c|c|c|c|c|c|c|}
\hline Sample & $\begin{array}{l}\text { Thickness } \\
/ \mu m\end{array}$ & $\begin{array}{l}\text { Grammage } \\
/ \mathrm{g} / \mathrm{m}^{2}\end{array}$ & $\begin{array}{l}E^{M D} \\
/ \mathrm{MPa}\end{array}$ & $\begin{array}{l}\sigma_{\max }^{M D} \\
/ \mathrm{MPa}\end{array}$ & $\varepsilon_{\max }^{M D}$ & $\begin{array}{l}E^{C D} \\
/ \mathrm{MPa}\end{array}$ & $\begin{array}{l}\sigma_{\max }^{C D} \\
/ \mathrm{MPa}\end{array}$ & $\varepsilon_{\max }^{C D}$ \\
\hline E0 & 238 & 166 & $5132[4800]$ & $56.1[64]$ & $0.027[0.03]$ & $3728[3100]$ & $39.1[41]$ & $0.035[0.03]$ \\
\hline $\mathrm{E} 0 *$ & 214 & 161 & 2113 & 46.3 & 0.056 & 2216 & 37.5 & 0.076 \\
\hline E10 & 231 & 173 & $4125[2100]$ & $60.8[44]$ & $0.051[0.06]$ & 1975 & 33.3 & 0.073 \\
\hline E15 & 234 & 172 & 2493 [1900] & $47.2[41]$ & $0.081[0.08]$ & 2082 & 31.5 & 0.075 \\
\hline E20 & 251 & 178 & $2624[1900]$ & $42.8[37]$ & $0.071[0.085]$ & - & - & - \\
\hline F0 & 244 & 171 & $5439[5200]$ & $63.3[71]$ & $0.032[0.03]$ & 3143 & 30.6 & 0.036 \\
\hline $\mathrm{F} 0 *$ & 206 & 156 & 2850 & 58.3 & 0.051 & 1252 & 24.8 & 0.082 \\
\hline F10 & 245 & 173 & $4205[3000]$ & $72[60]$ & $0.053[0.05]$ & $1877[1500]$ & $29.0[25]$ & $0.080[0.09]$ \\
\hline $\mathrm{F} 15$ & 264 & 172 & $3064[1900]$ & $54.6[40]$ & $0.066[0.07]$ & 1566 & 26.3 & 0.084 \\
\hline $\mathrm{F} 20$ & 251 & 178 & $1840[1300]$ & $44.6[38]$ & $0.095[0.09]$ & 1302 & 23.2 & 0.090 \\
\hline
\end{tabular}

* indicates samples with restrained drying. Values without brackets are from standard tensile test. Values within brackets corresponds to the samples used in this study

with a similar set up. The camera came calibrated from the supplier and the integration curve for temperatures between -10 and $50{ }^{\circ} \mathrm{C}$ was used. The emissivity of the paperboard was assumed to be 0.92 . The infra-red images were captured with a frame rate of $32 \mathrm{~Hz}$. Hence, 96 pictures could be captured if the strain at break was $5 \%$. The IR camera resolution was $640 \times 512$ but the resolution of the sample in it self was roughly $200 \times 400$ pixels. From these pictures the heat radiation in different positions could be observed. In Fig. 4, two typical IR pictures of the E20 sample, unstrained and prior to failure, can be seen. In the picture it can be observed that bands with increased temperature appear in the sample prior to break. From the pictures the average

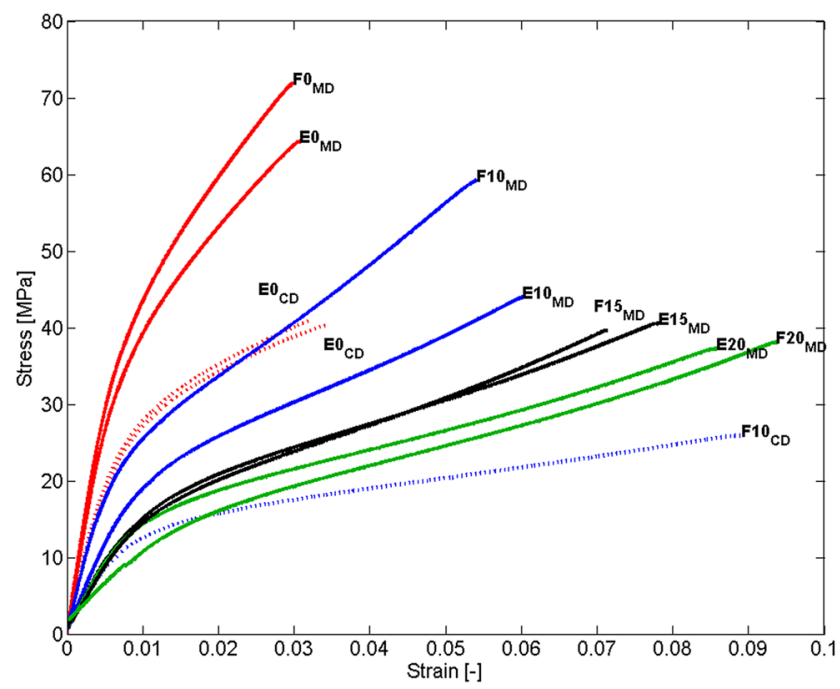

Fig. 2 Stress-strain curves for the samples used for thermography temperature of the sample was calculated as the average of all pixels within an area of roughly $47 \times 95 \mathrm{~mm}^{2}$, this area varied a bit from sample to sample as it was set manually, but as much as possible of the unstrained sample was used, see Fig. 4(a). The size and position of the measuring box remained the same during testing, as seen in Fig. 4(b). From this data the standard deviation of the temperature was also calculated. To avoid unnecessary disturbances the surrounding of the sample was screened with material that had low IR reflectance.

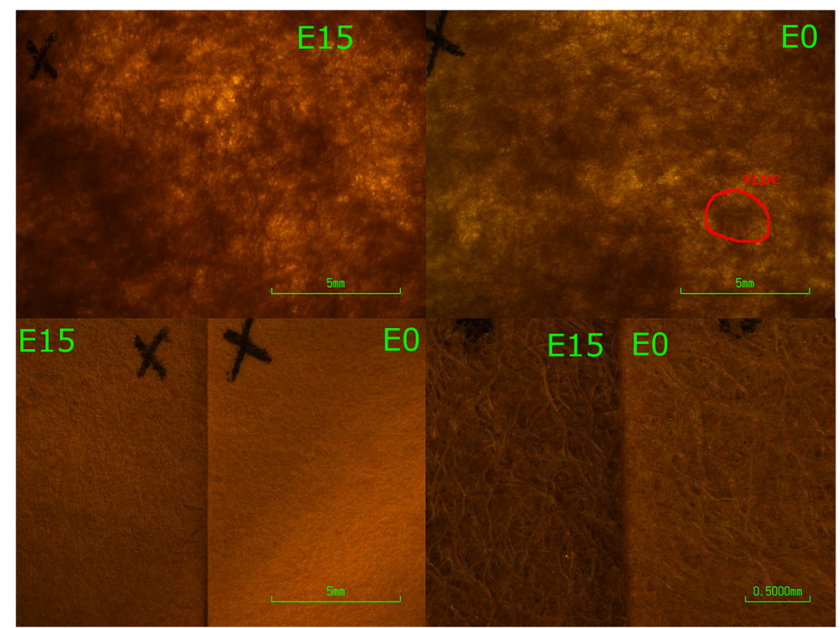

Fig. 3 Close ups of papers E0 and E15. Top pictures are backlit to emphasize the formation, flocs are clearly visible. Note the similarities between the two papers. Bottom left shows E0 and E15 next to each other, this time in direct light. Bottom right shows the a zoomed in view of the same boards. The compaction of the fibres is visible in the E15 paper which is shown as increased roughness 
Fig. 4 Example termographs of sample E20, unstrained (a) and prior to break (b), with measuring boxes used for temperature calculations

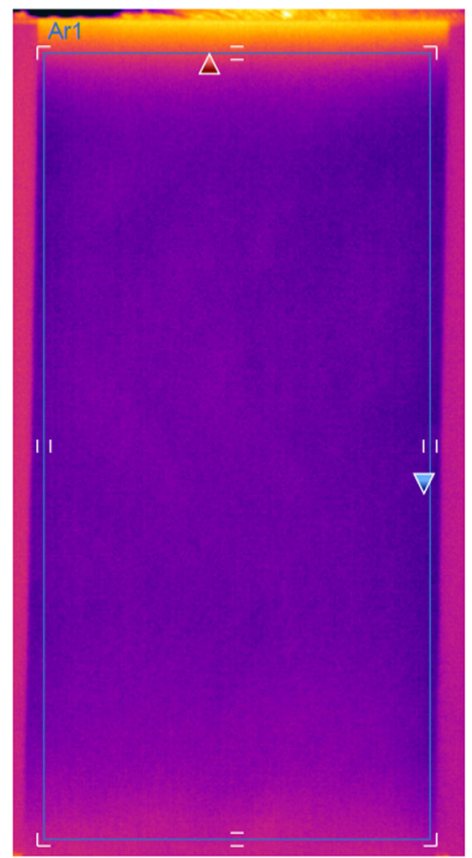

(a)

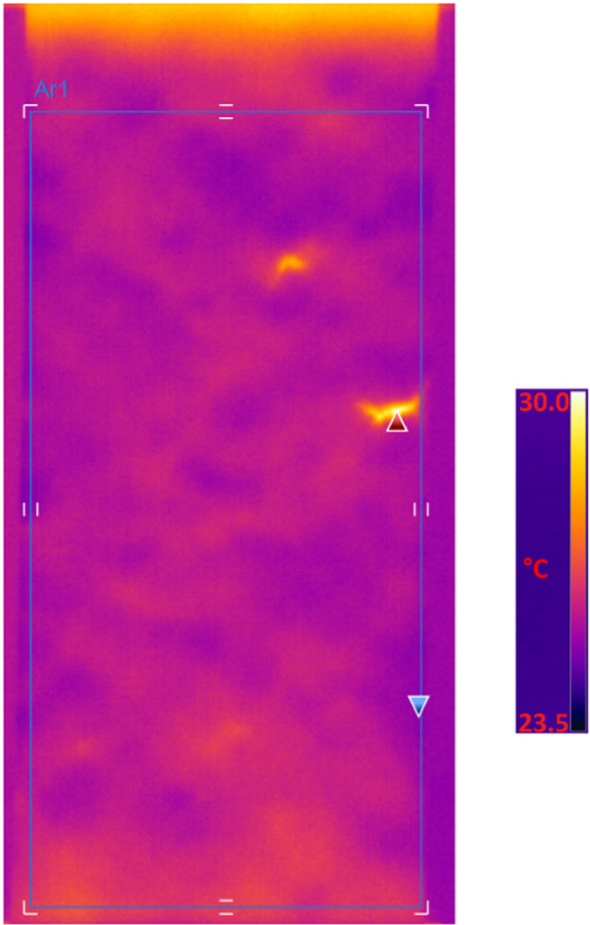

(b)
Digital image correlation The digital image correlation was done using stereo cameras in a commercial system, Aramis $^{\circledR}$ (GOM mbH, Braunschweig Germany). The system was chosen based on previous work, Hagman and Nygårds [18], but a single camera system would have been equally adequate, since the movement was mainly in the MD-CD plane. Prior to testing, a random speckle pattern was printed onto the paper samples using a standard office printer. Images was captured at $5 \mathrm{~Hz}$ and then analysed using the provided software. Details and technical information about the DIC set up and analysis can be found in Table 2. The strain fields presented in this work show the in-plane strain in the test direction.

Formation assessment The optical formation of the papers used was assessed using a Creo, iQsmart ${ }^{3}$ scanner. The scanner worked by transmitting light through the paper and measuring the translucency. Although the exact grammage variations were not calibrated the gray scale images could be used to identify flocs and other density variations in the paper. This made visual comparisons between the formations and other measurement methods possible. Further it was possible to use this data as a basis for property variations in the FEM analysis.

Finite element model Finite element simulations were performed in ABAQUS 6.12 (Dassault Systèmes Simulia
Corp.). The model of the test piece was simulated fully $(50 \mathrm{~mm} \times 100 \mathrm{~mm})$ using $167 \times 333$ block elements (C3D20) in one layer. In the model the bottom edge was fixed in the length direction, while the top edge moved 10 $\mathrm{mm}$. Both edges were able to move freely in the width and out-of-plane direction, as well as able to rotate in the out-ofthe plane direction. The boundary conditions were chosen to mimic the actual test.

To represent the elastic-plastic behavior of the papers, an orthotropic elastic model was used, together with an anisotropic Hill yield criterion with isotropic hardening. The advantage with this model is that it is a built in material model in Abaqus, which has previously been used by Huang and Nygårds [19, 20] with good accuracy. The material was defined by 9 elastic constants, 6 yield stresses in the primary and shear directions as well as a linear hardening which was determined by the hardening in the MD. To account for the heterogeneuos properties of the paper, the yield strength and hardening were also dependent on a spatial field, where each node were assigned a scalar value between 0 and 1 . The value in each individual node was based on the formation map. This was done by analyzing the gray level in the formation measurments at the site corresponding to the node location. Since the node coordinates and the pixel coordinates of the picture mismatched, an approximation had to be made. Hence the gray value of the pixel corresponding to the node position was chosen. 
Table 2 Technical information about the DIC system

\begin{tabular}{ll}
\hline Parameter & Setting \\
\hline Technique: & Stereo Image Correlation \\
Cameras: & EDS Vosskhler (Osnabrück, Germany) Model CCD-1300QB \\
Lens: & Schneider Kreuznach (Hauppauge, NY) 2.8/50, 16 mm \\
Sensor/digitization: & $1280 \times 1024,8$-bit \\
Camera Noise(\% of range): & $0.5 \%$ \\
Lighting: & Ambient white light \\
Lens/Imaging distance: & 5 mm, 70 cm to specimen, 30 cm between cameras \\
Pixel to mm conversion: & 1 pixel $=0.09$ mm \\
Software: & Aramis 6.1 \\
ROI: & $112 \mathrm{~mm} \times 89$ mm \\
Subset, step: & 15,13 \\
Shape function, accuracy, residual: & Bilinear, 0.04 pixels, 20 gray levels \\
Displacement Resolution: & $6.8 \mu m$ \\
Strain Window: & $3 \times 3$ \\
Strain Resolution: & $190 \mu \varepsilon$ \\
\hline
\end{tabular}

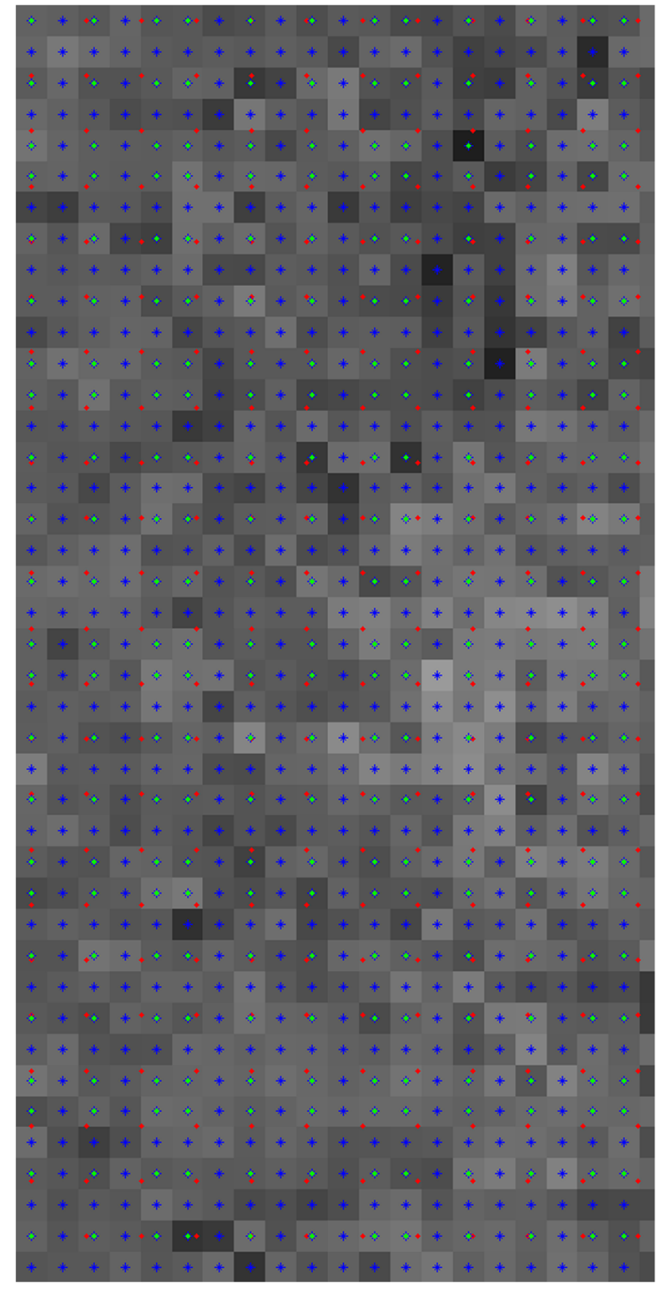

Fig. 5 Illustration of how the node values were chosen to represent formation. The node coordinates are shown in red, the center point of the pixels in blue and the chosen center points in green
This is illustrated in Fig. 5, where the red dots correspond to node locations, the blue spots indicate the middle of the pixels, and the green spots indicate the pixel that was chosen. This method was a bit rough, as some pixels are ignored. An illustration of the formation using only the selected pixels compared with the original picture can be found in Fig. 6(a). The resemblance were deemed to be good enough. Histograms of the distributions of the different graylevels, Fig. 6(b), are the same before and after the operation. The material data that was used in the simulations can be found in Table 3. The data was selected to resemble paper E15; the in-plane moduli of elasticity chosen close to the measured value, the ZD modulus was approximated to a tenth of the CD modulus. The shear moduli was not measured, but set to resemble those used in previous work [19]. The in-plane Poisson's ratios were approximated to be 0.3 based on previous work, e.g. Huang and Nygårds [19, 20]. The out-of-plane Poisson's ratio for paper is still under investigations, with reports of small and even negative values, e.g. Stenberg and Fellers [21]. Since it was judged to have small impact for these simulations it was set to zero. The determination of the plastic behaviour is discussed in the results section.

\section{Results}

In Table 1, the tensile test data for the uncreped and the creped sample were presented. From the table it was evident that there was a large difference between the freely dried papers compared with the paper with constrained drying. The freely dried samples had lower elastic modulus and higher strain at break than the samples with constrained 

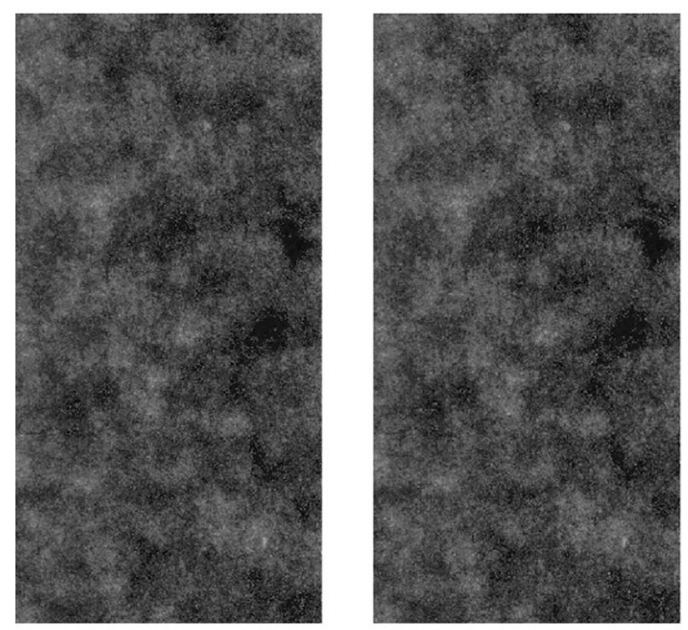

(a)
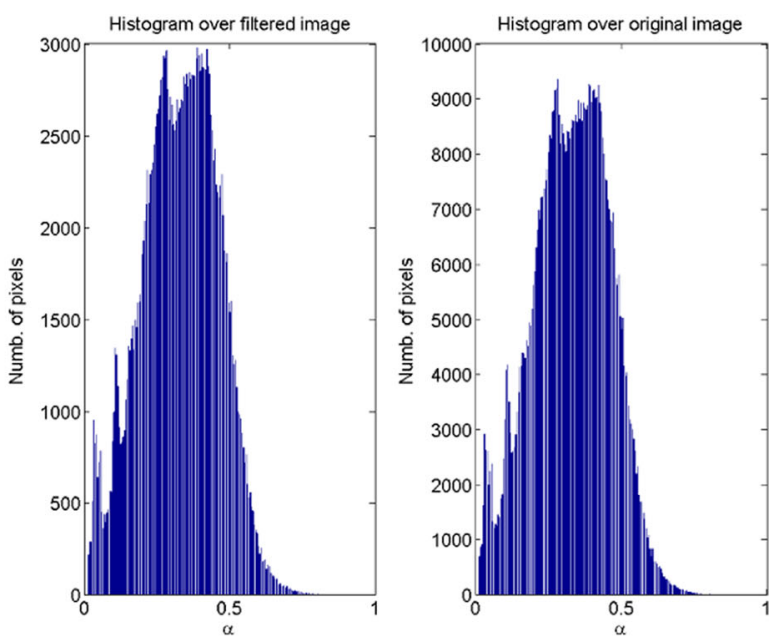

(b)

Fig. 6 Original formation pattern of a E15 sample (a-left), and modified pattern used as base for FEM-simulations (a-right). b Histograms of the distribution of the $\alpha$ parameter from the pictures

drying. Creping of the papers contributes to the same effects; lower modulus and higher strain at break. Overall it could be observed that the modulus of the creped papers approached the freely dried samples. This was especially apparent for CD specimens that had been creped in the MD. Freely dried samples will be compacted due to dried in stresses. This is because the fibres shrink during drying, and can hence consolidate the network. It is a similar mechanism that is activated during creping. The creped samples will have a shrinkage that comes from both free drying and compaction during creping.
Thermography results Due to the high frame rate a large number of pictures were captured for each strain test. From these pictures the average temperature of the sample and the deviation could be calculated using the measuring box, as shown in Fig. 4. In three figures example graphs of the stress (Fig. 7), normalized average temperature, i.e. $\frac{\text { Av. temp. }}{\text { Av. temp. } t=0}$ (Fig. 8), and normalized standard deviation, i.e. Std. Dev. of temp.
Std. Dev. of temp are presented. The standard deviation was the deviation of the temperature inside the measuring box at each given time. In the same way, the average temperature was the average temperature inside the box at each given time. The graphs

Table 3 Material parameters used as base in the FEM simulations

\begin{tabular}{|c|c|c|c|c|c|c|}
\hline \multicolumn{2}{|l|}{ Elastic } & \multicolumn{3}{|c|}{ Plastic Hardening } & \multicolumn{2}{|l|}{ Yield criteria } \\
\hline Param. & Value & $\sigma_{M D}[\mathrm{MPa}]$ & $\varepsilon^{p}$ & Field value $(\alpha)$ & Potential Dir. & $\sigma_{d i r}^{y} / \sigma_{m d}^{y}$ \\
\hline$E_{m d}$ & $2500 \mathrm{MPa}$ & 16.50 & 0 & 0.5 & $c d$ & 0.355 \\
\hline$E_{c d}$ & $2000 \mathrm{MPa}$ & 47.00 & 0.062 & 0.5 & $z d$ & 0.355 \\
\hline$E_{z d}$ & $200 \mathrm{MPa}$ & & & & $m d c d$ & 0.8 \\
\hline$v_{m d c d}$ & 0.3 & & & & $m d z d$ & 0.207 \\
\hline$v_{m d z d}$ & 0 & & & & $c d z d$ & 0.151 \\
\hline$v_{c d z d}$ & 0 & & & & & \\
\hline$G_{m d c d}$ & $1000 \mathrm{MPa}$ & & & & & \\
\hline$G_{m d z d}$ & $60 \mathrm{MPa}$ & & & & & \\
\hline$G_{c d z d}$ & $60 \mathrm{MPa}$ & & & & & \\
\hline
\end{tabular}

The data was chosen to resemble the E15 paper 
Fig. 7 Stress - strain. Top frame contains curves for paper E, bottom frame contains curves for paper $\mathrm{F}$
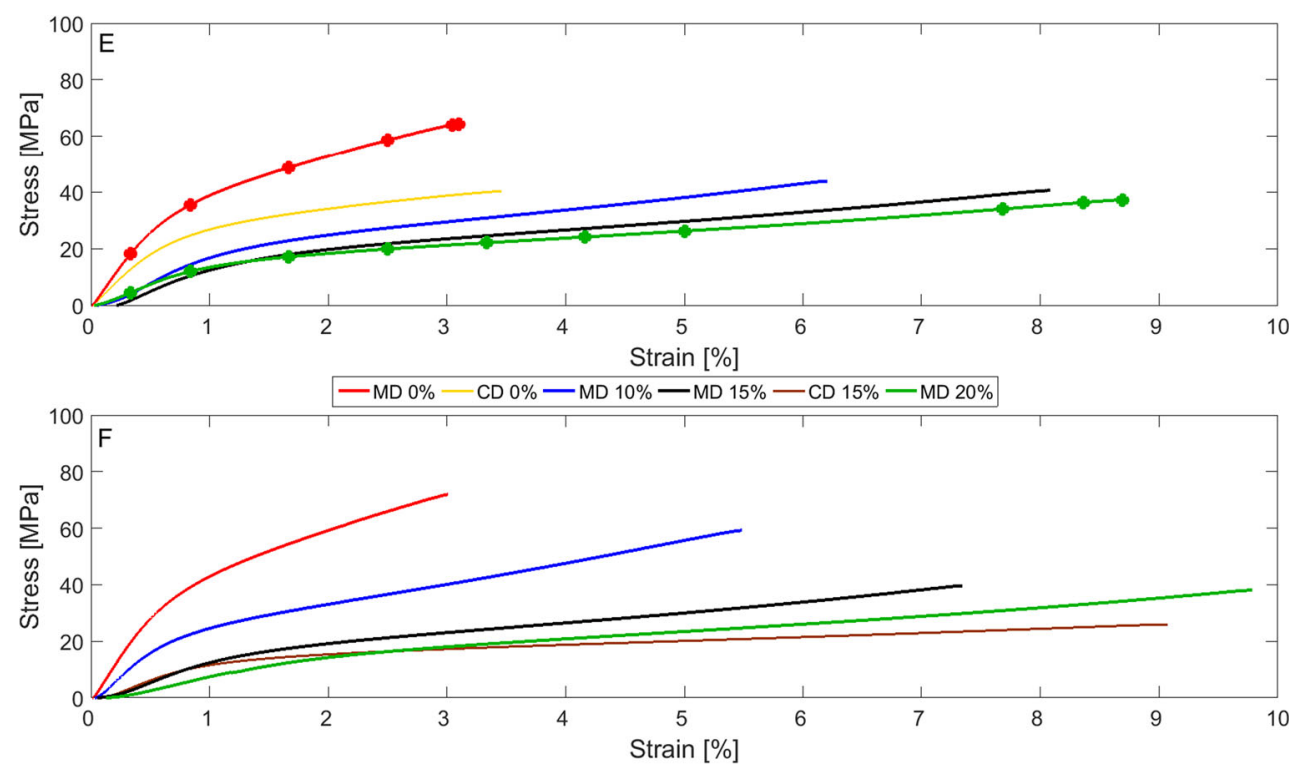

for the different papers have been separated into individual plots ( $\mathrm{E}$ above, $\mathrm{F}$ below). Thermographical pictures from two series, E0 and E20, have been selected and are shown in Fig. 10. The pictures were extracted at times indicated by the dots in Fig. 7. The two papers were chosen since they represent the extreme cases, no creping and the maximum creping.

From the data in Fig. 8 it can be seen that; the average temperature of the sample droped in the elastic regime of the test, and then rose when plastic deformation was initiated. Both the drop and the first part of the rise were parabolic in shape, but the rise became linear as time progressed. The temperature drop regime corresponded well with the elastic part of the stress time, i.e. strain, curve. This temperature drop for the elastic part has been observed previously by e.g Yamauchi et al. [7], as well as Dumbleton et al. [13].

The standard deviation of normalized temperature typically decreases initially and starts to slowly increase as plastic deformation is initiated, c.f. Fig. 9. The drop was due to the fact that there were less noise as the sample started to cool down. The increased standard deviation in the plastic part of the test was associated to the deformation patterns that emerged once the sample left the elastic region, see Fig. 10.

The deformation patterns in Fig. 10 were caused by energy emitted from the sample. The patterns reflect the papers inhomogeneous formation. Note how the average temperature increases when the patterns appear. This energy
Fig. 8 Average temperature strain. Temperature was measured in the square shown in the pictures in Fig. 4 and normalized with the value at time zero, denoted C. Top frame contains curves for paper $\mathrm{E}$, bottom frame contains curves for paper $\mathrm{F}$
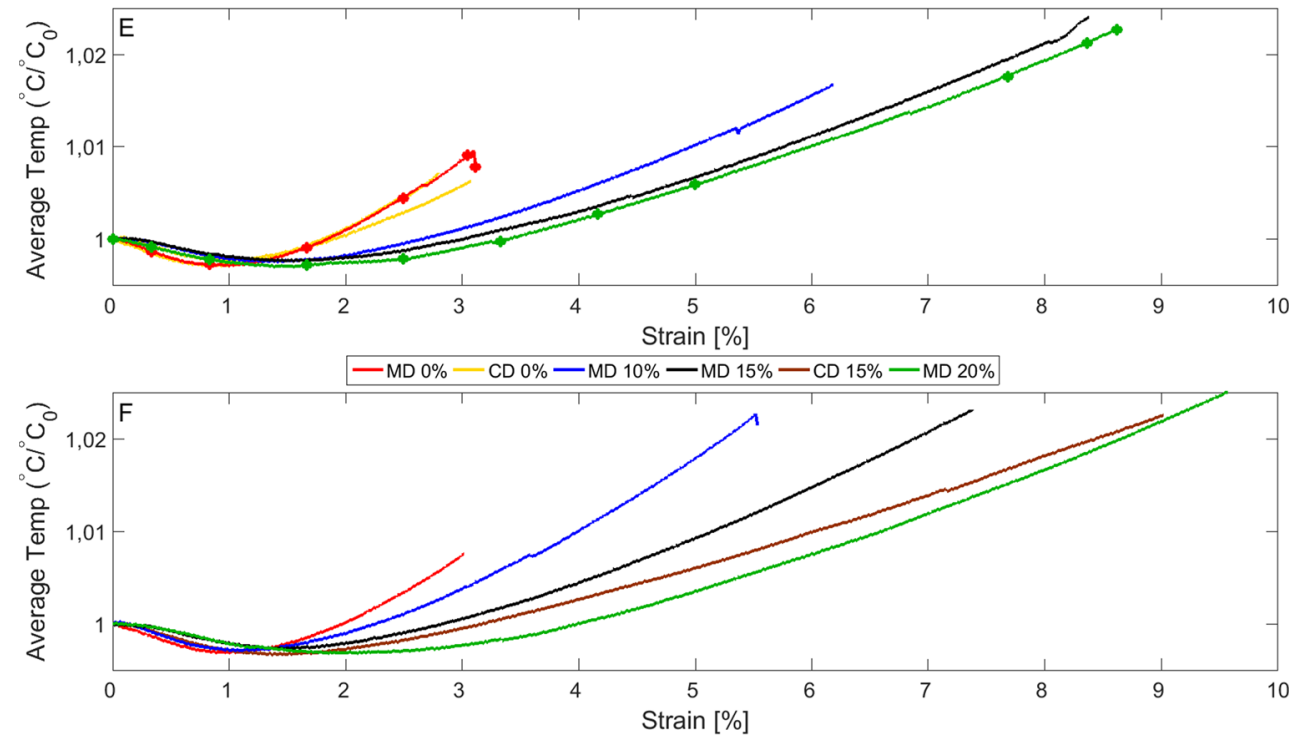
Fig. 9 Standard deviation strain. Standard deviation was measured in the square shown in the pictures in Fig. 4 and normalized with the value at time zero, denoted C. Top frame contains curves for paper E, bottom frame contains curves for paper $\mathrm{F}$
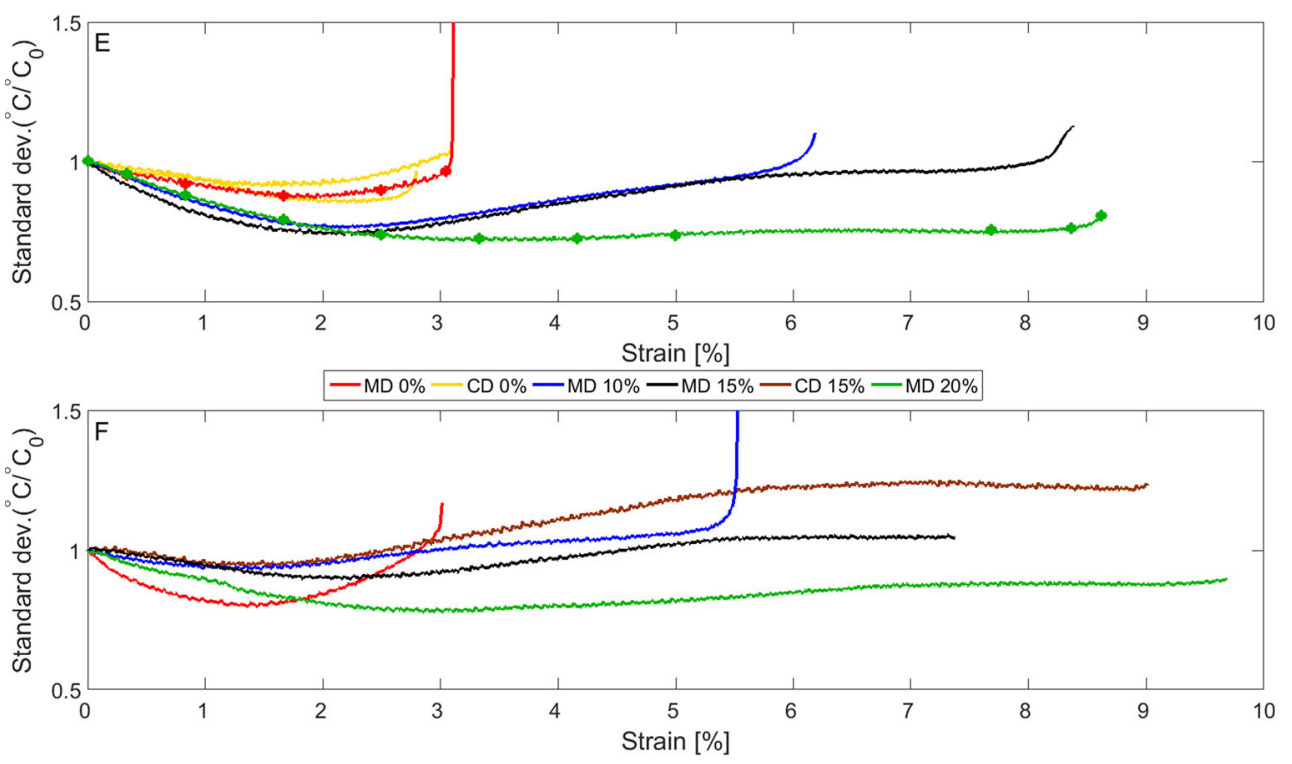

was assumed to be due to bond breaking, fibre movement and fibres deforming plastically as well as water expulsion as the whole sample starts to deform plastically. It was worth noting that it was possible to predict the rupture starting point by identifying the spots with the highest heat signature, but it was not possible to predict the cracking path from these signatures. The patterns that emerges were reminiscent of the shear bands seen in metals. The conclusion was that the areas with much emitted energy should be the areas that suffer from large local deformation, to test this heat signatures and the local strain field extracted using DIC will be compared.

Thermographical pictures from testing of two papers with different crepe levels (E0 and E20) were shown in Fig. 10. In both papers, similar deformation pattern emerged. The main difference was that the pattern was delayed in the creped sample. The stress, normalized average temperature and normalized standard deviation vs. strain from those series as well as two intermediate creping levels were presented in the Figs. 7-9. Looking at the graphs it was first of all obvious that the more creped papers possess higher strain-ability. From the average temperatures it can be seen that the first decrease in temperature was fairly similar for all creping levels. The temperature rise was similar but elongated for creped samples, the linear behaviour was again due to the constant rate of the displacement. The behaviour of the standard deviation changes from being a parabola for the uncreped samples to have a much flatter slope until the rapture. From this it can be argued that a seemingly elastic strain overlaid the regular behaviour. Such a strain could be the result of the deformation caused by the creping. It is not possible to determine the exact nature of this deformation using the thermographs. Two possible deformation mechanisms during creping would be either microwrinkeling of the sheet or that the fibres were slightly rotated from the machine direction. The latter explanation also takes into account that the behaviour becomes more CD like when the paper was creped, which was observed, but was not explicitly shown here. As seen in the bottom right part of Fig. 3, at least the surface fibres have been
Fig. 10 Thermal images from the points indicated by dots in the graphs in Fig. 7, top row E0, bottom row E20. Since the pictures where croped manually, they all have slightly different dimensions, but have here been scaled to the same hight, in reality the pictures should get taller when going from left to right, compare to Fig. 11
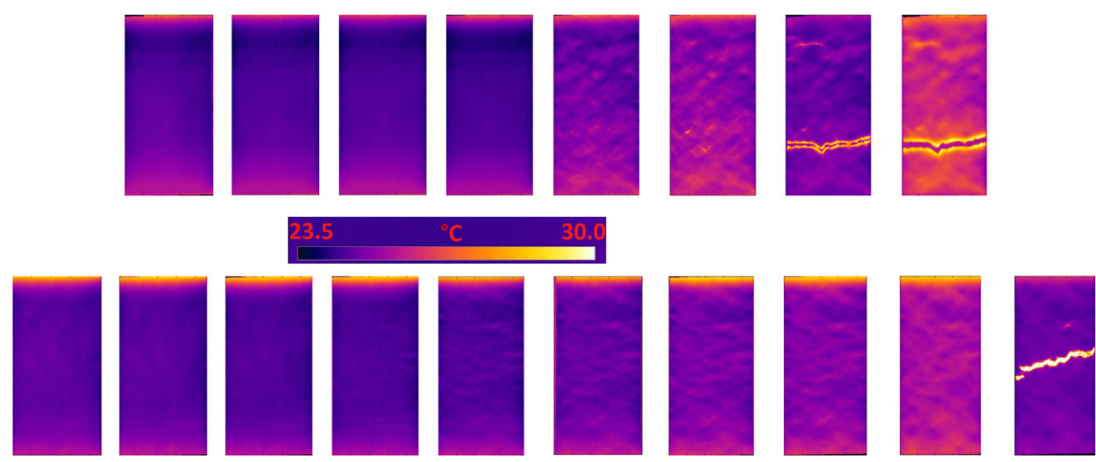
Fig. 11 Top row, thermographs of E15 from times indicated in Fig. 13. Bottom row the total inplane strain in the test direction as evaluated by DIC for the same times. Note that the strain scale is changing from picture to picture to contrast patterns. This makes comparisons between different pictures difficult. The DIC pictures are mirrored since they were captured from the opposite side of the sample compared to the thermographs
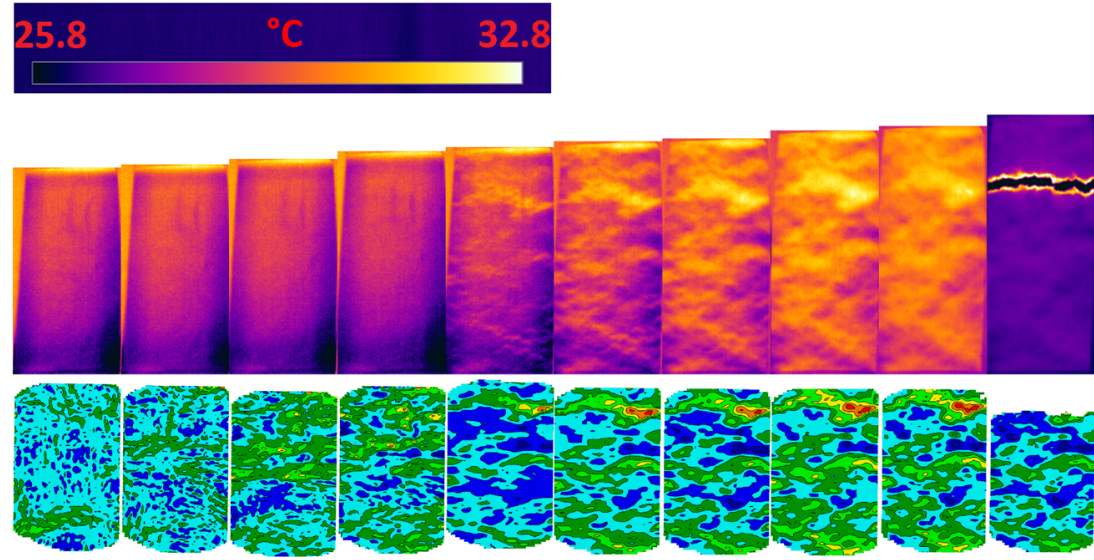

affected by the creping. The recovery of this overlaid deformation was linear to the stress, and is not entirely recovered until after the yield points in the weaker parts of the material. Since the creping was done in the machine direction the major effect was expected in that direction and this also seems to be the case, only minor effects were seen in the CD.

Thermography vs. DIC In Fig. 11, thermographs of a sample (E15) at different global straining levels, are compared with the strain fields from DIC at the corresponding times. in total 10 strain levels have been chosen from the beginning of the test until rupture. For each strain level two pictures are shown, in the top row the thermographs and in the bottom row the DIC strain fields. The strain fields depict the total in-plane strain, compared with the unstrained sample, in the test direction. An enlarged version of the pictures from one strain level are shown together with an overlaid picture in Fig. 12. The strain field was manually fitted over the thermal pictures, based on approximation of which part of the sample that was photographed by the DIC cameras. From these pictures we can observe that the hot zones in the thermograph correlate very well with the zones that have undergone large straining. This was a further indication that the energy released was due to the local plastic deformation in the sample.

To further support this idea, a comparison between average and variation from both the thermographical pictures and DIC pictures in Fig. 12 has been made, see Fig. 13. Both strain and temperature behaves in a similar manner. The average temperatures increased linearly, see Fig. 13(b), as can be expected since the test was displacement controlled with a fixed rate. However the fact that the standard deviations of temperature and local strain track each other, see Fig. 13(c), was a clear indication that it was the same processes that gave rise to both signals. This was further strengthened when the standard deviations were plotted against each other, as done in Fig. 13(d). A linear behaviour was observed in the plastic region. The strain/heat patterns that were seen, were on the same scale as the flocs that
Fig. 12 Left-to-right, thermal results, mirrored results from DIC, showing cumulated strain in test direction, with scale and the same picture overlaid over thermal results. Note how areas with small strain corresponds to cooler areas in the thermal photo. Both pictures at a global strain of approx $6.5 \%$

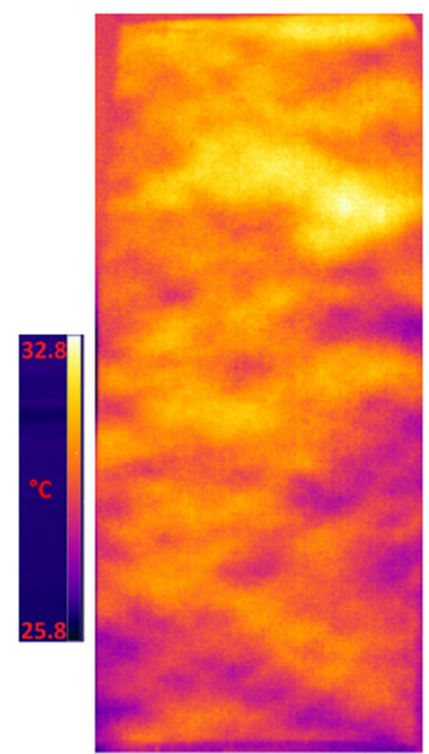




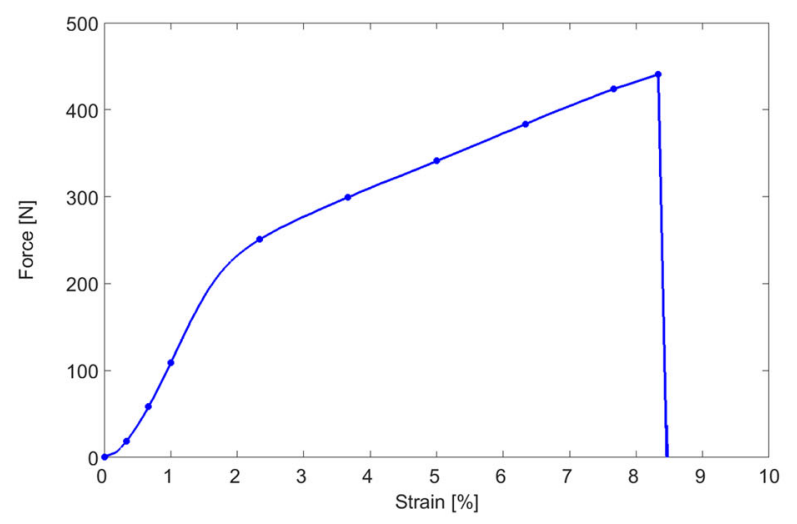

(a)

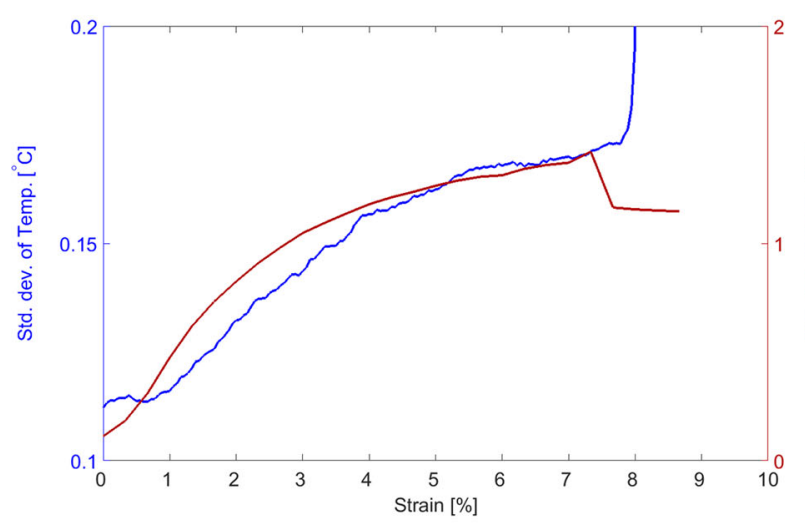

(c)

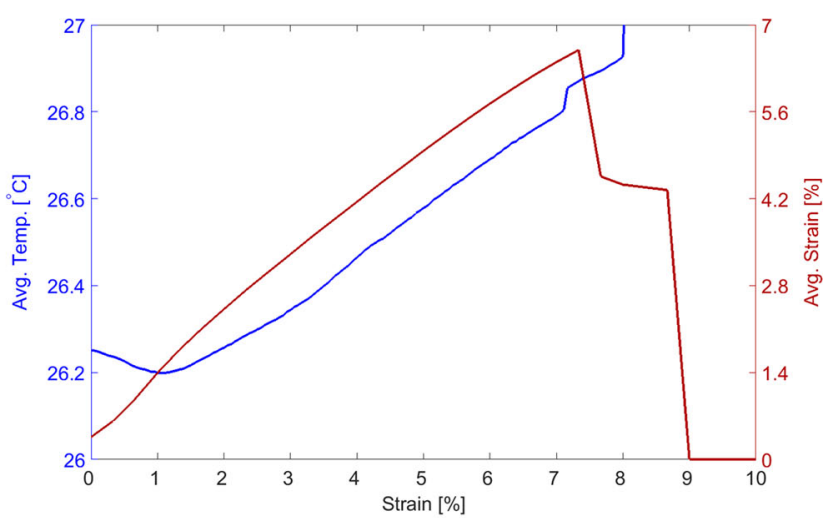

(b)

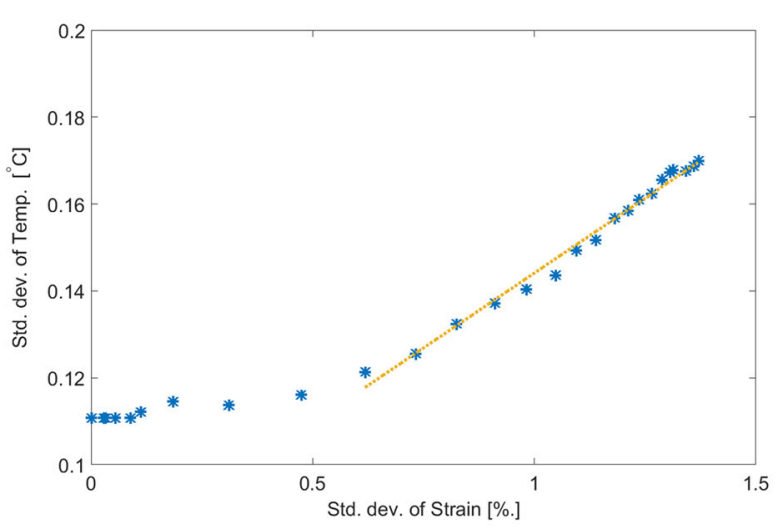

(d)

Fig. 13 Comparison of thermographs and DIC for paper E15; (a) Stress - Strain with time of pictures marked as dots, (b) Average temperature and local strain data - Global Strain, (c) Standard deviation of temperature and local strain - Global Strain, (d) Standard deviation of temperature - Standard deviation of strain

are seen in paper which makes a comparison between the formation of the paper and the heat streaks interesting.

Formation vs. strain pattern In Fig. 14, the thermograph images and the formation was compared in a similar way as in Fig. 12. Although these pictures are harder to compare, as strain patterns emerges after the yield point and the formation pictures are from unstrained samples, it was still quite apparent that the formation maps and strain patterns were similar. In the overlay picture the thermograph has been evenly scaled with the global strain in the length direction. Although such a scaling was far from perfect, it still makes the comparison easier. The comparison indicates that deformation takes place in areas with lower density, which can be identified from the formation pictures. This then contributed to the evolution of the inhomogeneous deformation patterns.

In order to validate the idea that formation contributes to the evolved deformation patterns FEM simulations were performed. To determine how the material behavior varied with varying density several approaches was numerically examined. In the end, the material was varied accordingly; the elastic modulus was held constant, the yield stresses, $\sigma_{y}$, and hardening moduluses were altered, and the ultimate strength was constant. The avarage behavior of the
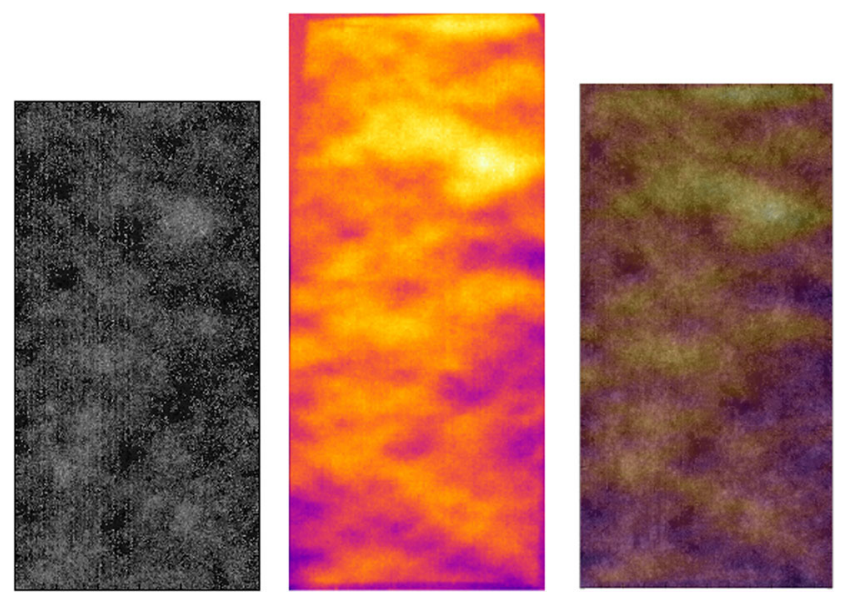

Fig. 14 From left to right, formation, thermal image (at $6 \%$ global strain) and thermal image scaled and overlaid on formation image. Note how dense areas correspond to low temperature areas 
Fig. 15 Left side, FEMprediction, based on formation. Middle DIC image. Right side thermal image. All pictures have a global strain of about $6.5 \%$

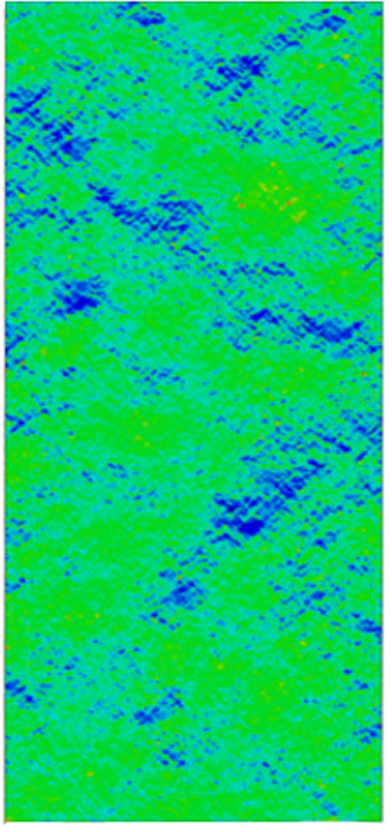

paper was first set, based on the measured stress-strain curves. This resulted in the material properties shown in Table 3, where the determination of the properties followed the same procedure as previously suggested by Huang and Nygårds [19]. The plastic data was thereafter altered in a range $\pm 50 \%$ which were found to produce a strain pattern of magnitudes similar to what was observed from the DIC measurements.

The correlation was confirmed when comparing the FEM simulated sample, which was based on the formation, with the thermograph and DIC picture as seen in Fig. 15. Apart from showing that it was the low density areas that were deformed, this gives a hint of the predictive potential of the FEM method together with the formation in-data.
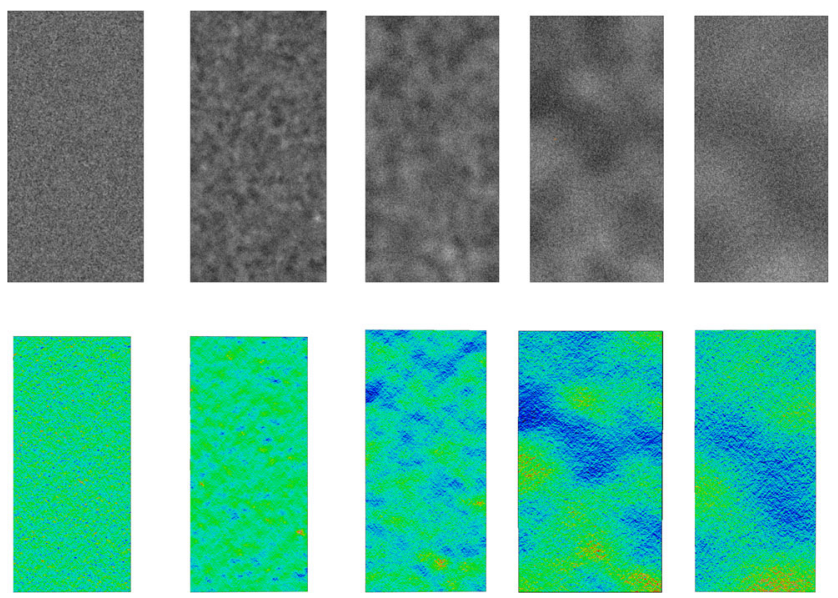

Fig. 16 Top row, computer generated formations. Bottom row, the local strains (in the test direction) at around $6 \%$ global strain

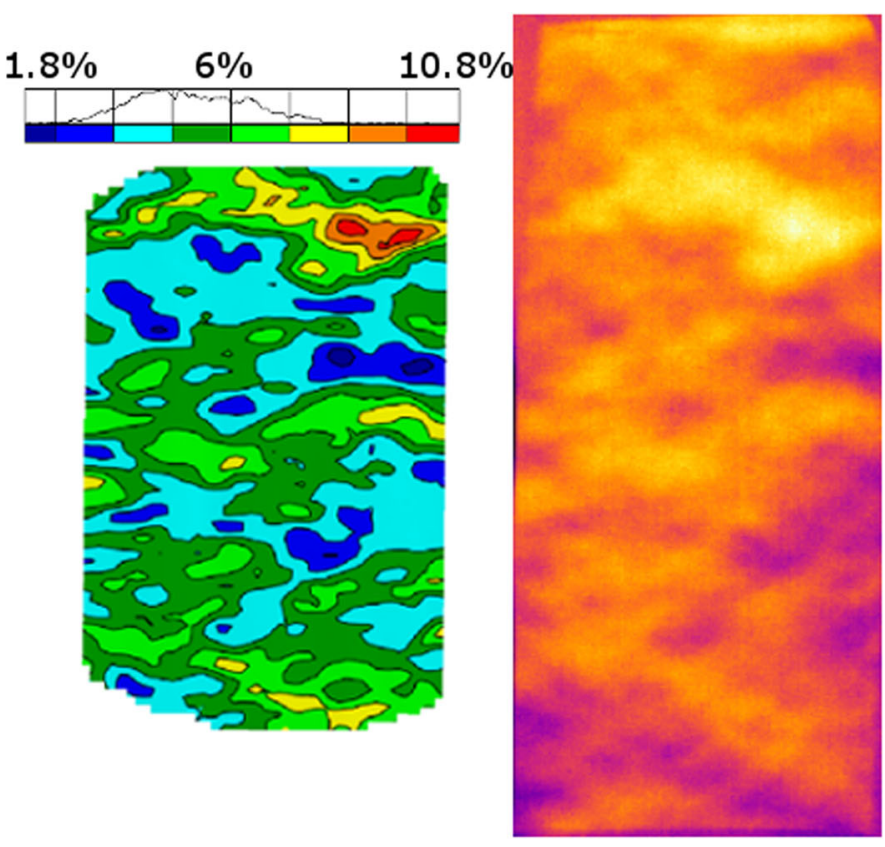

Using this insight, a small test where 5 computer generated boards with different formation was simulated. The five boards, labelled Gen. 1 to Gen. 5, were generated to go from a totally random distribution (Gen.1) to small flocs and then to larger flocs (Gen. 5). The algorithm by which these boards were generated can be found in the appendix. The generated papers can be seen in Fig. 16 together with their response at $6 \%$ global strain. The $\alpha$ distribution for the papers are shown in Fig. 17(a). The predicted stress-strain response of the papers, together with the response from the simulation based on the real formation and a homogeneous paper are found in Fig. 17(b). In addition, the behaviour of the standard deviation of temperatures from the boards are shown in Fig. 17(c). The simulated force displacement curves for all generated boards were fairly similar. All were weaker than both simulations based on the real sample and that based on a homogeneous paper.

The standard deviation shows clearer differences between the papers, dividing them into three groups; Gen. 4 and Gen. 5 with a high deviation that was steadily increasing, Gen. 3 and Gen. 1, with a medium deviation that flattens out somewhat before "rupture", and Gen. 2 that has the lowest deviation which actually decreases before "rupture". The paper based on the real formation behaved in a way that was similar to Gen. 2. From the deviation, and the pictures from the simulations it was clear that a coarse formation was bad for the stress strain behaviour, causing large zones where straining occurred. Additionally, a paper that does not have clear flocs, but still has random properties also becomes sensitive to its weakest spot. Boards with a fine formation, performed best in the simulations. 
(a)

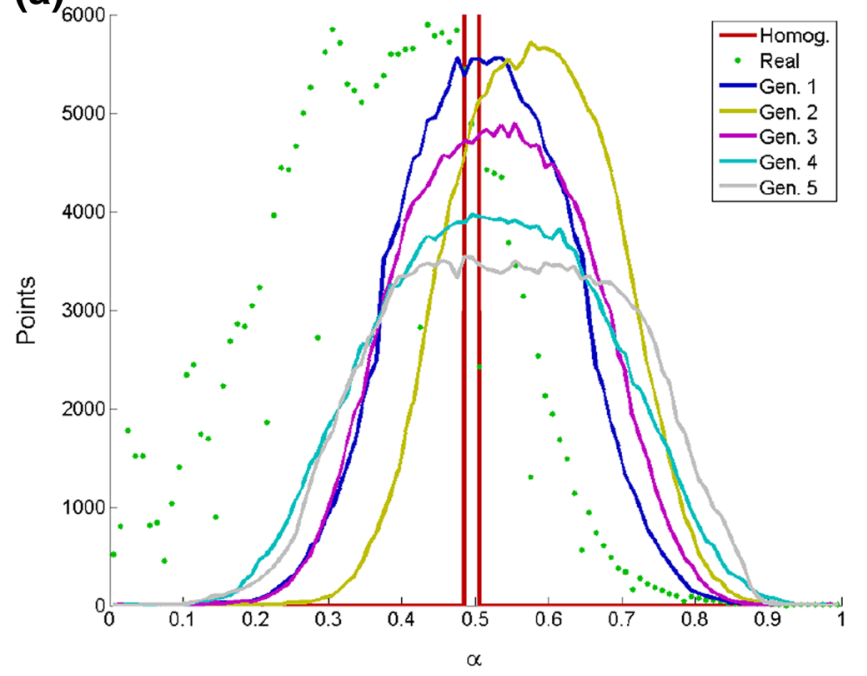

(b)

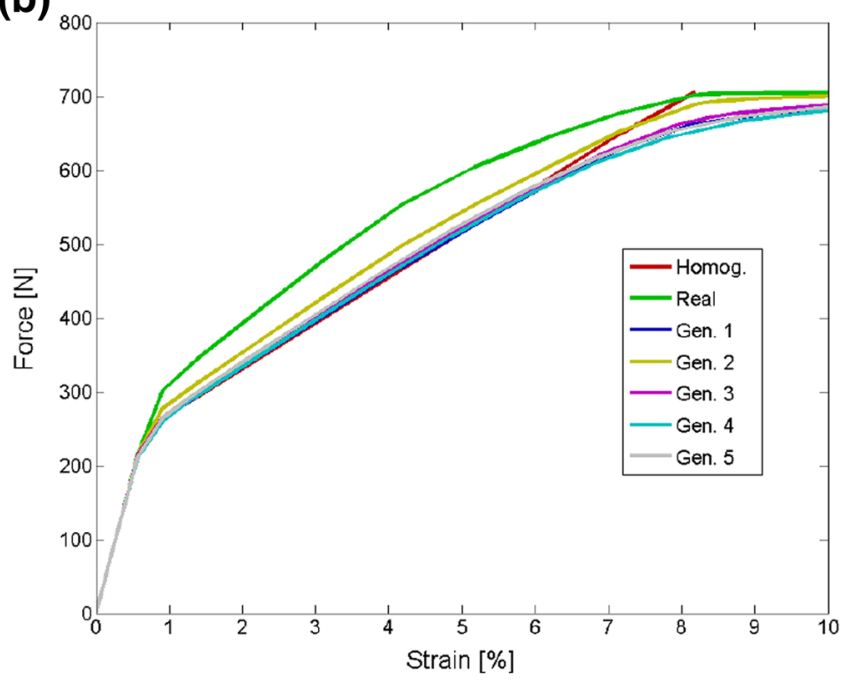

(c)

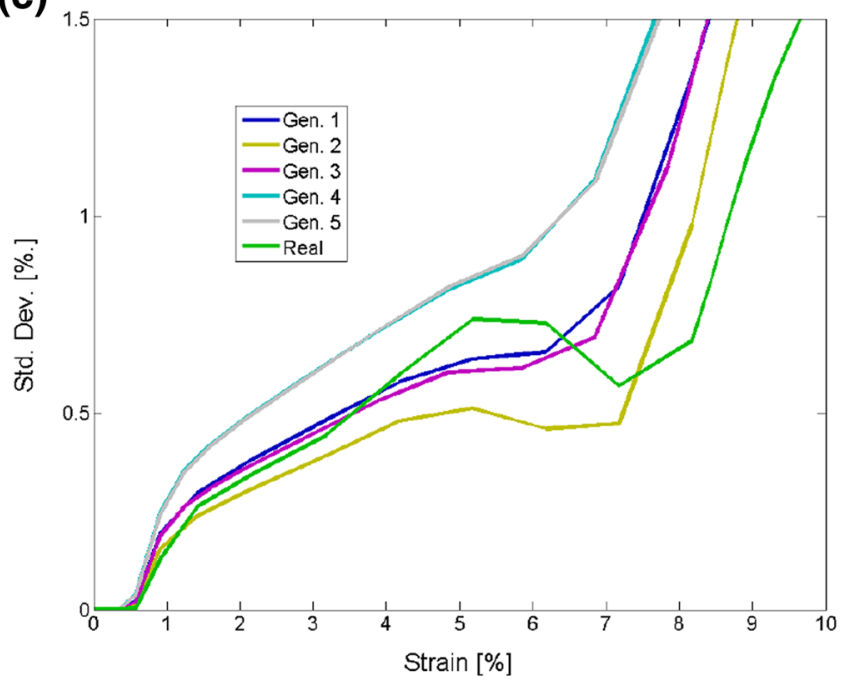

Fig. 17 Comparison curves for the five generated formations as well as the measured one and a homogeneous sample; (a) The alpha distributions, (b) Force - Strain, (c) Standard deviation - Strain

\section{Summary and Discussion}

Thermography The time and space resolution of the temperature data allows for a more careful analysis of the behaviour of the patterns that emerges. This analysis can be split into two parts. One visual, where the patterns that emerge can be compared with patterns from other tools, such as DIC and formation. The other part was an analysis of the pattern itself. This in turn can be split into typical behaviours, such as preferred directions of the streaks, and statistical analysis of the pattern. Although it was hard to conclude anything about the preferred directions of the streak pattern, they appeared roughly 40 degrees from the machine direction, independent of the straining. The statistical measure that was chosen was to study the standard deviation from the mean temperature in a measuring box. A problem with this technique was that the box was stationary, i.e. a certain part of each picture was chosen, this means that when the sample stretched, part of it moved out of the measuring area, while other parts move into it. The measuring box was placed near the bottom of the sample, which limited the inflow of new material into the measuring box. An increased standard deviation indicates that the difference in temperature, i.e. strain level, between the high and low intensity regions has increased. In the first part of testing the standard deviation decreased, this was most likely due to an increased signal from the whole sample during elastic loading. The whole sample starts cooling evenly, and the noise 
that was present in the beginning was drowned in this signal. When the sample starts to emit energy the difference between different parts of the sample comes into play, different amount of energy was released, patterns emerged and the variation started to increase.

\section{Thermography compared to other measurements As} shown in the results section the thermographic images correlate well with results from both DIC measurements and the formation. Since the thermographic measurements show that the sample was fairly homogeneous during the elastic straining it was interesting to compare with the DIC at the same time. The aim was to see if any information was hidden in the temperature data. The DIC images from the elastic region showed random strain variation, both in space and time, which was in contrast to the clear patterns that emerges in the plastic region. Similar observations have been made in previous work [18, 22]. Since both the local strain and temperature patterns in the plastic region correlates well with the formation of the sample, this indicates, somewhat unintuitively that the formation plays a minor role in the initial response of the paper.

The comparison of the thermographs and the formation was a bit harder to do than that with the DIC, since the formation, as mentioned, is only measured in the undeformed state. Despite this obstacle it was still possible to see that the heat pattern correlates well with the formation, especially since such a correlation seems very reasonable. The FEM simulation further enhanced the correlation. It should be pointed out that the FEM simulations made in this article should be viewed as merely rudimentary proof of concepts. As such the material data used are approximate at best. What were carefully considered in the FEM simulations were the conditions for how the plastic behaviour was initiated and described.

Regarding the mechanisms behind the increased strainability in creped papers no trends related to the spatial distribution of the strain pattern, or underlying formation, could be discerned. What was observed was that the creped samples behave differently when looking at the development with time. Looking at the stress strain curves for different levels of creping it can be seen that the samples yield at approximately the same level of strain, the more creped samples somewhat later than the uncreped. The pictures support this, in that the patterns are present from approximately the same times, once again somewhat later for the creped samples. Same trends can be seen in the average temperature curve. This makes the mechanism suggested in the results section, i.e. an overlaid linear recoverable deformation, quite likely. The standard deviation of temperature curves further supports this, as the deviation for the highly creped papers was almost constant for a long time when the paper had left the elastic zone, this should be the case if the heat was generated by two sources, one that acts on the whole paper and one that was localized. When the paper was strained in the weaker part it generated heat in those parts, but if the paper at the same time generates heat evenly, the difference was kept constant.

An increased homogeneity of the paper should nevertheless increase the strain at break, since it is the most locally strained part of the paper that initiates rupture, leaving other sections only partially strained compared to their maximum potential. This in turn results in a waste of fibres from the paper manufacturers perspective. It should however be noted that a perfectly homogeneous sheet would be more sensitive to disturbances. Thus there might be an optimum with small evenly distributed flocs.

\section{Conclusions}

The heat pattern generated during plastic straining was proportional to the local straining, which in turn was dependent on the underlying formation.

From the heat generated it was possible to predict where the crack causing rupture will initiate, but not how the crack itself will propagate.

This heat pattern only occurred during plastic straining which indicates that formation played a minor role in the initial response of the paper. The elastic behaviour seemed mostly due to effects of the drying.

Although the FEM in this work is rudimentary the results are encouraging, and it seems plausible that the strain behaviour in paper can be predicted using FEM based on the formation.

Regarding the mechanism at work when the strain at break was increased by creping the papers. There was no changes in the underlying formation of the paper or other signs of homogenization. Instead the increased strain at break seemed to be caused by an overlaid deformation that could be recovered during straining.

An increased homogeneity of the paper should nevertheless increase the strain at break, since it is the most locally strained part of the paper that initiates rupture, leaving other sections only partially strained compared to their maximum potential.

Acknowledgments The authors would like to acknowledge the VINNOVA Excellence Centre BiMaC Innovation for the financial support. The help from Elisabet Horvath and Jonas Sundström at Innventia during production and creping of the papers was highly appreciated. Moreover, the help by Andreas Gabrielsson and Aron Tysén at Innventia with the IR camera was also greatly appreciated.

Open Access This article is distributed under the terms of the Creative Commons Attribution 4.0 International License (http:// creativecommons.org/licenses/by/4.0/), which permits unrestricted use, distribution, and reproduction in any medium, provided you give 
appropriate credit to the original author(s) and the source, provide a link to the Creative Commons license, and indicate if changes were made.

\section{Appendix: Board Generation Algorithm}

The generated formations for board Gen. 1 to 5 were acquired in the following way using Matlab ${ }^{\circledR}$ 2010a (The Mathworks inc.).

1. An empty matrix with each element corresponding to a node location is created.

2. Until the matrix is filled repeat:

(a) An empty element is chosen randomly.

(b) The mean value of the non-empty elements in a box of $2 \mathrm{X}$ elements is calculated. If $\mathrm{X}$ is bigger then the distance to the wall, the box is cut of at the wall.

(c) The element is assigned a value that is the mean value + a random value between -0.5 and 0.5 .

3. The the absolute value of the matrix minimum is added to the matrix which is then divided by the new maximum value, resulting in all values being between 0 and 1 .

The different boards where produced by choosing different values of $X$ and in that way controlling how large the irregularities should be. The values chosen was: 1, 5, 10, 20 and 40. Which should be compared to the size of the matrix that was $335 \times 667$ elements.

\section{References}

1. Stenberg N (2003) A model for the through-thickness elasticplastic behaviour of paper. Int J Solids Struct 40(26):7483-7498

2. Nygårds M (2008) Experimental techniques for characterization of elastic-plastic material properties in paperboard. Nordic Pulp Paper Res J 23(4):432-437

3. Kerekes RJ, Soszynski RM, Doo T The flocculation of pulp fibres

4. Marais A, Magnusson MS, Joffre T, Wernersson ELG, Wagberg L (2014) New insights into the mechanisms behind the strengthening of lignocellulosic fibrous networks with polyamines. Cellulose 21(6):3941-3950

5. Magnusson MS, Zhang X, Östlund S (2013) Experimental evaluation of the interfibre joint strength of papermaking fibres in terms of manufacturing parameters and in two different loading directions. Exper Mech 53(9):1621-1634

6. Hyll C, Vomhoff H, Nygårds M (2012) Analysis of the plastic and elastic energy during the deformation and rupture of a paper sample using thermography. Nordic Pulp Paper Res J 27(2):329334

7. Yamauchi T, Okumura S, Noguchi M (1993) Application of thermography to the deforming process of paper materials. J Mater Sci 28(17):4549-4552

8. Tanaka A, Kettunen H, Yamauchi T (2003) Comparison of thermal maps with damage revealed by silicone impregnation. Paperi ja puu 85(5):287-290

9. Tanaka A, Yamauchi T (2000) Deformation and fracture of paper during the in-plane fracture toughness testing - examination of the essential work of fracture method. J Mater Sci 35(7):1827-1833

10. Tanaka A, Otsuka Y, Yamauchi T (1997) In-plane fracture toughness testing of paper using thermography. Tappi J 80(5):222226

11. Tysen A, Vomhoff H (2015) Method for the quantification of inplane drying non-uniformity. Nordic Pulp Paper Res J 30(2):286295

12. Ebeling KI Distribution of energy consumption during the straining of paper

13. Dumbleton DP, Kringstad KP, Soremark C (1973) Temperature profiles in paper during straining. Svensk papperstidning

14. Yamauchi T, Murakami K (1994) Observation of deforming process of a poorly formed papersheet by thermography. Sen'I Gakkaishi 50(9):424-425

15. Chrysochoos A, Huon V, Jourdan F, Muracciole J-M, Peyroux R, Wattrisse B (2010) Use of full-field digital image correlation and infrared thermography measurements for the thermomechanical analysis of material behaviour. Strain 46(1):117-130

16. Cholewa N, Summers PT, Feih S, Mouritz AP, Lattimer BY, Case SW (2015) A technique for coupled thermomechanical response measurement using infrared thermography and digital image correlation (tdic). Exper Mech:1-20

17. Korteoja MJ, Lukkarinen A, Kaski K, Gunderson DE, Dahlke JL, Niskanen KJ (1996) Local strain fields in paper. Tappi J 79(4):217-223

18. Hagman A, Nygårds M (2012) Investigation of sample-size effects on in-plane tensile testing of paperboard. Nordic Pulp Paper Res J 27(2):295-304

19. Huang H, Nygårds $M$ (2010) A simplified material model for finite element analysis of paperboard creasing. Nordic Pulp Paper Res J 25(4):505-512

20. Huang H, Nygårds M (2011) Numerical and experimental investigation of paperboard folding. Nordic Pulp Paper Res J 26(4):452467

21. Stenberg N, Fellers C (2002) Out-of-plane poisson's ratios of paper and paperboard. Nordic Pulp Paper Res J 17(4):387-394

22. Considine JM, Scott CT, Gleisner R, Zhu JY (2005) Use of digital image correlation to study the local deformation field of paper and paperboard. In: Transactions of the 13th fundamental research symposium, pp 613-630 